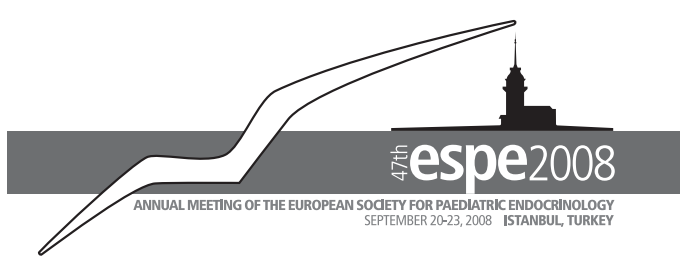

\title{
47th Annual Meeting of the European Society for Paediatric Endocrinology (ESPE)
}

İstanbul, Turkey, September 20-23, 2008

\section{Abstracts}

Guest Editor

Atilla Büyükgebiz, İstanbul, Turkey

Programme Organising Committee

J.-C. Carel, France, Chair

A. Büyükgebiz, Turkey, President

S. Cianfarani, Italy, CPC Chair

L. Sävendahl, Sweden, Member

E. Malecka-Tendera, Poland, Member

J. Lebl, Czech Republic, Member

H. Krude, Germany, Member

Local Organising Committee
A. Abacı, İzmir
F. Çizmecioglu, Kocaeli
A. Akıncı, Malatya
H. Günöz, İstanbul
A. Bideci, Ankara
M. Berberoglu, Ankara
A. Büyükgebiz, İstanbul
N. Kandemir, Ankara
A. Kemal Topaloglu, Adana
O. Evliyaoglu, Kırıkkale
B. Dündar, Isparta
Ö. Tarım, Bursa
B. Özkan, Erzurum
O. Ercan, İstanbul
D. Gökşen, İzmir
P. Cinaz, Ankara
E. Böber, İzmir
S. Darcan, Izmir
E. Çetinkaya, Ankara
S. Kurdoglu, Kayseri
F. Darendeliler, İstanbul 


\section{This publication was sponsored by}

\author{
Eli Lilly and Company \\ Ferring Pharmaceuticals \\ Ipsen \\ Merck Serono \\ Novo Nordisk \\ Pfizer Endocrine Care \\ Sandoz International
}

S. Karger

Medical and Scientific Publishers Basel $\cdot$ Freiburg $\bullet$ Paris $\bullet$ London New York $\cdot$ Bangalore $\cdot$ Bangkok Shanghai $\cdot$ Singapore $\cdot$ Tokyo $\cdot$ Sydney
Disclaimer

The statements, options and data contained in this publication are solely those of the individual authors and contributors and not of the publisher and the editor(s). The appearance of advertisements in the journal is not a warranty, endorsement, or approval of the products or services advertised or of their effectiveness, quality or safety. The publisher and the editor(s) disclaim responsibility for any injury to persons or property resulting from any ideas, methods, instructions or products referred to in the content or advertisements.

Drug Dosage

The authors and the publisher have exerted every effort to en sure that drug selection and dosage set forth in this text are in accord with current recommendations and practice at the time of publication. However, in view of ongoing research, changes in government regulations, and the constant flow of information relating to drug therapy and drug reactions, the reader is urged to check the package insert for each drug for any change in indications and dosage and for added warnings and precautions. This is particularly important when the recommended agent is a new and/or infrequently employed drug.
All rights reserved

No part of this publication may be translated into other languages, reproduced or utilized in any form or by any means, electronic or mechanical, including photocopying, recording, microcopying, or by any information storage and retrieval system, without permission in writing from the publisher or, in the case of photocopying, direct payment of a specified fee to the Copyright Clearance Center (see 'General Information')

(c) Copyright 2008 by S. Karger AG

P.O. Box, CH-4009 Basel (Switzerland)

ISBN 978-3-8055-8620-7

Electronic production of the abstract book by

pharma service - a business unit of documediaS $\mathrm{GmbH}$ Günther-Wagner-Allee 13, D-30177 Hannover (Germany) www.pharmaservice.de

Printed by

Lindendruck Verlagsgesellschaft $\mathrm{mbH}$

Fössestrasse 97A, D-30453 Hannover (Germany) www.lindendruck.de

\section{KARGER}




\section{Contents}

\section{Plenary Lectures}

\begin{tabular}{|c|c|c|c|c|}
\hline 1 & PL1 & Genes and Environment & $1-2$ & Saturday, September 20 \\
\hline 1 & PL2 & From Gene to Disease & 3-4 & Sunday, September 21 \\
\hline & PL3 & ESPE Award Session \& Activities 1 & no abstracts & Monday, September 22 \\
\hline 2 & PL4 & Inheritance, Development and Behavior: Lessons from Aromatase Mutations & $5-6$ & Monday, September 22 \\
\hline \multirow[t]{2}{*}{2} & PL6 & ESPE Award Session \& Activities 2 (Henning Andersen Prize Presentations) & $9-10$ & Tuesday, September 23 \\
\hline & \multicolumn{2}{|c|}{ Symposia } & & \\
\hline 5 & S3 & Evolution for the Paediatric Endocrinologist & $17-19$ & Saturday, September 20 \\
\hline 5 & S4 & Symposia Istanbul - Bridge the Gap in Child Health Between East and West & $20-22$ & Saturday, September 20 \\
\hline 6 & S5 & Long Term Consequences of Early Feeding & $23-25$ & Monday, September 22 \\
\hline 7 & S6 & New Trends in Thyroid Screening & $26-28$ & Monday, September 22 \\
\hline 7 & S7 & Genetic Networks and Development & $29-31$ & Monday, September 22 \\
\hline 10 & \multicolumn{2}{|c|}{ New Technologies } & & \\
\hline 11 & NT1 & New Technologies 1 & $41-42$ & Sunday, September 21 \\
\hline \multirow[t]{2}{*}{11} & NT2 & New Technologies 2 & $43-44$ & Tuesday, September 23 \\
\hline & \multicolumn{2}{|c|}{ ESPE Working Groups } & & \\
\hline 13 & WG1 & Joint Session of the ESPE Bone Club and the ESPE Growth Plate Working Group & $45-48$ & Saturday, September 20 \\
\hline \multirow[t]{2}{*}{14} & WG2 & ESPE Bone Club & $49-50$ & Saturday, September 20 \\
\hline & WG3 & ESPE Growth Plate working Group & no abstracts & Saturday, September 20 \\
\hline$\overline{14}$ & WG4 & $\begin{array}{l}\text { ESPE Turner Syndrome Working Group } \\
\text { Turner Syndrome (TS) and the Cardiovascular System }\end{array}$ & $51-54$ & Saturday, September 20 \\
\hline 15 & WG5 & $\begin{array}{l}\text { ESPE Obesity Club The Metabolic Syndrome in the Paediatric Age Range - } \\
\text { Towards International Consensus }\end{array}$ & $55-58$ & Saturday, September 20 \\
\hline 27 & FC6 & Diabetes and Insulin Resistance & $98-103$ & Sunday, September 21 \\
\hline 29 & $\mathrm{FC7}$ & Disorders of Sex Development & 104-109 & Sunday, September 21 \\
\hline 31 & FC8 & Genetics of Grow th & $110-115$ & Sunday, September 21 \\
\hline 32 & FC9 & Central Weight Regulation & $116-121$ & Sunday, September 21 \\
\hline 34 & FC10 & Top Rated Basic Abstracts & $122-126$ & Sunday, September 21 \\
\hline 36 & FC11 & Bone & 127-132 & Monday, September 22 \\
\hline 38 & FC12 & Top Rated Clinical Abstracts & $133-140$ & Monday, September 22 \\
\hline 40 & FC13 & Genetics of Hormone Excess & $141-146$ & Tuesday, September 23 \\
\hline 42 & FC14 & Turner and Noonan Syndrome & $147-152$ & Tuesday, September 23 \\
\hline
\end{tabular}


Poster Presentations

\begin{tabular}{|c|c|c|c|c|}
\hline 45 & P1-d1 & Adrenal 1 & $153-162$ & Sunday, September 21 \\
\hline 48 & P1-d1 & Bone 1 & $163-174$ & Sunday, September 21 \\
\hline$\underline{52}$ & $\mathrm{P} 1-\mathrm{d} 1$ & Pancreas 1 & $175-191$ & Sunday, September 21 \\
\hline 57 & $\mathrm{P} 1-\mathrm{d} 1$ & Thyroid 1 & $192-206$ & Sunday, September 21 \\
\hline 61 & $\mathrm{P} 1-\mathrm{d} 2$ & Obesity and Fat 2 & $207-234$ & Monday, September 22 \\
\hline 69 & $\mathrm{P} 1-\mathrm{d} 2$ & Reproductive Endocrinology 1 & $235-256$ & Monday, September 22 \\
\hline 76 & P1-d3 & GH Treatment 2 & $257-272$ & Tuesday, September 23 \\
\hline 81 & $\mathrm{P} 1-\mathrm{d} 3$ & Growth 2 & $273-306$ & Tuesday, September 23 \\
\hline 91 & P2-d1 & GH Physiology & $307-317$ & Sunday, September 21 \\
\hline 94 & P2-d1 & GH Treatment 1 & $318-355$ & Sunday, September 21 \\
\hline 106 & P2-d1 & Growth 1 & $356-391$ & Sunday, September 21 \\
\hline 117 & P2-d1 & Obesity and Fat 1 & $392-455$ & Sunday, September 21 \\
\hline 136 & P2-d2 & Adrenal 2 & $456-490$ & Monday, September 22 \\
\hline 146 & P2-d2 & Others & $491-502$ & Monday, September 22 \\
\hline 149 & P2-d2 & Perinatal Endocrinology & $503-514$ & Monday, September 22 \\
\hline 153 & P2-d2 & Sex Differentiation & $515-529$ & Monday, September 22 \\
\hline 158 & P2-d2 & SGA & $530-542$ & Monday, September 22 \\
\hline 162 & P2-d2 & Thyroid 2 & $543-590$ & Monday, September 22 \\
\hline 176 & P2-d2 & Turner Syndrome & $591-605$ & Monday, September 22 \\
\hline 181 & P2-d3 & Autoimmunity & $606-615$ & Tuesday, September 23 \\
\hline 183 & P2-d3 & Bone 2 & $616-631$ & Tuesday, September 23 \\
\hline 188 & P2-d3 & Calcium & $632-644$ & Tuesday, September 23 \\
\hline 192 & P2-d3 & Endocrine Genetics & $645-665$ & Tuesday, September 23 \\
\hline 198 & P2-d3 & Pancreas 2 & $666-718$ & Tuesday, September 23 \\
\hline 214 & P2-d3 & Reproductive Endocrinology 2 & $719-750$ & Tuesday, September 23 \\
\hline \multicolumn{5}{|c|}{ Read by Title } \\
\hline 224 & $\mathrm{R}$ & Read by Title & $1-121$ & \\
\hline
\end{tabular}

$\begin{array}{ll}259 & \text { Abstract Reviewers } \\ 261 & \text { Author Index } \\ 277 & \text { Subject Index }\end{array}$

